\title{
EXPERIMENTAL EVIDENCE FOR BEAM-BEAM DISRUPTION AT THE SLC ${ }^{*}$
}

\author{
T. Barklow, G. Bower, F.J. Decker, C. Field, L.J. Hendrickson, T. Markiewicz, \\ D.J. McCormick, M. Minty, N. Phinney ${ }^{\#}$, P. Raimondi ${ }^{+}$, M.C. Ross, K.A. Thompson, \\ T. Usher, M.D. Woodley, F. Zimmermann', SLAC, Stanford, CA
}

\begin{abstract}
A significant luminosity enhancement from beam-beam disruption was observed for the first time during the 199798 run of the SLAC Linear Collider (SLC). Disruption, or pinch effect, is due to the decrease in effective beam size during collisions as each beam is focused by the field of the other beam. With beam intensities of $4.010^{10}$ per bunch, bunch lengths of $1.1 \mathrm{~mm}$, and horizontal and vertical beam sizes of $\sigma_{x}=1.5 \mu \mathrm{m}$ and $\sigma_{y}=0.65 \mu \mathrm{m}$, the luminosity increase from disruption was more than $100 \%$. Measured data rates as recorded by the SLD detector were in agreement with the theoretically calculated disruption enhancement.
\end{abstract}

\section{INTRODUCTION}

The beam-beam interaction has been studied extensively both for electron-positron storage rings and for linear colliders. During the collisions, each beam is focused by the collective electromagnetic fields of the other oppositely charged beam. In storage rings, this additional focusing causes the beam-beam tune shift which must be controlled so that the beams do not become unstable. For a linear collider, if the focal length is on the order of or less than the bunch length, the effective beam size shrinks during the collisions, increasing the luminosity. In principle, since the beams are discarded after colliding, one tries to maximize the beam-beam interaction and produce the largest luminosity enhancement.

The practical limit on the disruption value achieved may be set by the increase in outgoing emittance and the beamstrahlung radiation. The larger emittance of the disrupted beam may be difficult to capture and extract cleanly, causing backgrounds in the detector or problems with critical downstream diagnostics. For high energy colliders, the beamstrahlung radiation becomes significant and the particles which have radiated collide at lower energy than nominal. This smearing of the collision energy can degrade the quality of physics experiments. An additional practical problem is that the disruption severely distorts standard techniques for measuring the beam size at the interaction point such as beam-beam deflection

"Work supported by the U.S. Dept. of Energy under contract DE-AC0376SF00515

"Email: nan@slac.stanford.edu

'Present address: SL Division, CERN, CH1211 Geneva 23, Switzerland scans, making it difficult to properly characterize the beams. To extract the correct beam size, the effects of disruption must be included in the analysis.

\section{THEORETICAL FRAMEWORK}

The phenomenon of disruption was studied for round beams $\left(\sigma_{x} / \sigma_{y}=1\right)$ by Hollebeek in 1980 [1] and for flat beams $\left(\sigma_{x} / \sigma_{y}>>1\right)$ by Chen [2] and others. The strength of the effect is characterized by the disruption parameter, $\mathrm{D}_{\mathrm{x}, \mathrm{y}}$, for each plane which is the inverse focal length in units of the bunch length, $\sigma_{z}$.

$$
D_{x, y}=\frac{2 N r_{e} \sigma_{z}}{\gamma \sigma_{x, y}\left(\sigma_{x}+\sigma_{y}\right)}
$$

where $\sigma_{x, y, z}$ are the horizontal, vertical and longitudinal beam sizes, $\mathrm{N}$ is the bunch population and $\mathrm{r}_{\mathrm{e}}$ is the classical electron radius. The luminosity enhancement factor, $H_{D}$, is the increase in luminosity due to the change in beam size. For typical machine parameters, the beambeam interaction is non-linear and cannot be calculated analytically. Simulations must be used to model the behavior using programs such as ABEL [3] or more recently, Guinea Pig [4]. Chen and Yokoya [5] derived a parameterization of $\mathrm{H}_{\mathrm{D}}$ for the case of round beams which is given by the equation

$$
H_{D}=1+D^{1 / 4}\left(\frac{D^{3}}{1+D^{3}}\right)\{\ln (\sqrt{D}+1)+2 \ln (0.8 / A)\}
$$

where $D$ is the same for both planes and $A=\sigma_{z} / \beta^{*}$, the ratio of the bunch length to the $\beta$ function at the collision point. For SLC operation, the horizontal beam size is only a factor of two to three larger than the vertical beam size and is closer to a round beam than to the extreme flat beam limit. Chen [6] proposed that for such "quasiflat" beams, the luminosity enhancement scales as

$$
H_{D}=H_{D}^{1 / 2} H_{D}^{f(R)}
$$

where

$$
f(R)=\frac{1+2 R^{3}}{6 R^{3}}
$$

with $\mathrm{R}=\sigma_{x} / \sigma_{y}$ and where both $\mathrm{H}_{\mathrm{D} x}$ and $\mathrm{H}_{\mathrm{D} y}$ are approximated by the round beam parameterization given 
in equation (2). This formula has been used to predict the expected disruption enhancement at the SLC.

\section{SLC HISTORY}

Prior to the 1994-95 SLC run, the beam intensity was limited to less than $3^{*} 10^{10}$ particles per bunch by the onset of a microwave bunch lengthening instability in the damping rings. The instability was caused by the interaction of the beam with sharp transitions in the vacuum chamber diameter. For 1994, new low impedance vacuum chambers with smooth transitions were installed in both rings. There was also a major upgrade to the optics of the final focus to reduce contributions to the beam size from $3^{\text {rd }}$ order aberrations caused by the interleaved horizontal and vertical sextupoles in the SLC chromatic correction sections. These two upgrades combined with other improvements to the beam stability and tuning finally brought the SLC beam parameters into the regime where disruption could contribute to the luminosity. The predicted disruption enhancement was about $20 \%$. The parameters achieved in 1994-95 are listed in Table 1 along with those for 1996 and for the 1997-98 run.

For the short 1996 run, there were no major upgrades to the machine but the beam intensity was increased slightly and better emittance control resulted in smaller horizontal beam sizes. The predicted disruption enhancement was about $30 \%$. In both runs, only a small excess of luminosity was seen beyond what would be predicted for undisrupted beams, $5-10 \%$. At least two factors contributed to the discrepancy between predicted and observed luminosity enhancement. An analysis of the final optimization procedures at the interaction point (IP) indicated that the resolution was inadequate for micron size beams. Mistuning was estimated to degrade the luminosity by up to $30 \%$ [7]. After the 1996 run, it was also found that the bunch length had been much shorter than desireable for maximal disruption.

The primary evidence for disruption seen in this period was a distortion of the deflection angles during the beambeam deflection scans used to measure the beam size at the IP. If the fit to the deflection data assumed that the beams remained rigid throughout the collision, the vertical beam size would be overestimated by up to $25 \%$ [8]. A new fit had to be developed which took into account the change in beam size described by equation (3).

\section{1997-98 SLC RUN}

In the 1997-98 run, the SLC luminosity increased by more than a factor of three [9]. More than 350,000 $\mathrm{Z}^{0}$ events were recorded by the SLD detector, nearly double the total sample from all previous runs. A major contribution to this performance came from a significant disruption enhancement, typically $50-100 \%$, meaning that about half of the luminosity was due to disruption. The improvement was due almost entirely to changes in tuning procedures and reconfiguration of existing hardware with no major upgrade projects. Improved alignment and emittance tuning techniques throughout the accelerator resulted in minimal emittance growth from the damping rings to the final focus. In particular, a revised strategy for wakefield cancellation using precision beam size measurements at the entrance of the final focus improved emittance optimization. The final focus lattice was modified to provide stronger demagnification near the interaction point and to remove residual higher-order aberrations. Beam sizes as small as 1.5 by 0.65 microns were achieved at full beam intensity of $4 * 10^{10}$ particles per pulse. These parameters are shown in Table 1.

Table 1: Beam parameters at the SLC interaction point

\begin{tabular}{|c|c|c|c|}
\hline & 1994 / 95 & 1996 & 1997 / 98 \\
\hline$\left(10^{10}\right)$ & 3.5 & 3.7 & 4.0 \\
\hline$(\mu \mathbf{m})$ & $\begin{array}{ll}3.7 & 1.2 \\
\end{array}$ & $\begin{array}{ll}3.2 & 1.2 \\
\end{array}$ & $\begin{array}{ll}2.1 & 0.9\end{array}$ \\
\hline$(\mathbf{m m})$ & 1.0 & 1.0 & 1.1 \\
\hline$(\mu$ rad $)$ & $350 \quad 270$ & $350 \quad 270$ & $450 \quad 250$ \\
\hline $\mathbf{H}_{\mathrm{D}}$ & 1.2 & 1.3 & 2.1 \\
\hline L $\quad\left(10^{30} / \mathrm{cm}^{2} / \mathrm{sec}\right)$ & 0.75 & 1.0 & 2.8 \\
\hline
\end{tabular}

To eliminate the tuning problems encountered in previous runs, a novel 'dithering' feedback was implemented to perform the final beam optimization at the IP [10]. Five corrections must be routinely applied to each beam which include centering of the $x$ and $y$ beam waist positions, zeroing of the dispersion $\eta_{x}$ and $\eta_{y}$, and minimizing an $x-y$ coupling term. The feedback optimized a direct measure of the luminosity as a function of small changes in each parameter. By averaging over thousands of beam pulses, it was possible to improve the resolution by a factor of 10 . Care was also taken to optimize the longitudinal beam profile and bunch length in the linac using dedicated machine experiments [11].

\section{MEASUREMENT OF $\mathrm{H}_{\mathrm{D}}$}

The value of $H_{D}$ can be measured by comparing the luminosity as recorded by SLD to that which would be predicted for rigid beams without disruption given the observed SLC beam parameters. To improve statistics, the SLD luminosity was calculated from the number of $Z^{0}$ and Bhabha scattering events recorded in each half hour interval. The Bhabha cross section is 3.65 times the $Z^{0}$ production cross section at the mass of the $Z^{0}$. In any finite interval, some fraction of the beam pulses are not available for luminosity either because of machine protection or diagnostic tuning procedures. To compensate for the variable rate of these missing pulses, the raw Bhabha plus $Z^{0}$ event count must be scaled up to an 
equivalent $120 \mathrm{~Hz}$ rate, by dividing by the fraction of pulses with both beams in collision at the IP. The SLD fast data acquisition recorded beam intensities as well as signals from beamstrahlung and luminosity counters for every pulse at $120 \mathrm{~Hz}$. This data could then be used to accurately count pulses with beams in collision.

The calculation of SLC luminosity without disruption was based on the beam intensity for each pulse from the SLD $120 \mathrm{~Hz}$ data stream. The transverse beam overlap size was measured at least hourly with beam-beam deflection scans, where the fit to the deflection angle versus position data included a correction for the beam size change during collision. To improve the quality of these scans, the incoming beam trajectories are measured on each pulse and the fitted position of the beams at the IP is used to correct for pulse-to-pulse jitter. These trajectory measurements are also used to calculate the RMS jitter for the two beams in both planes and provide an estimate of the luminosity loss due to pulse-to-pulse motion. This correction factor, typically about $6 \%$, is applied to the calculated SLC luminosity. Figure 1 shows the measured disruption enhancement factor, $\mathrm{H}_{\mathrm{D}}$, for different values of the SLD luminosity. $\mathrm{H}_{\mathrm{D}}$ is the ratio of actual SLD luminosity to that predicted from the observed SLC beam parameters without disruption, as described above.

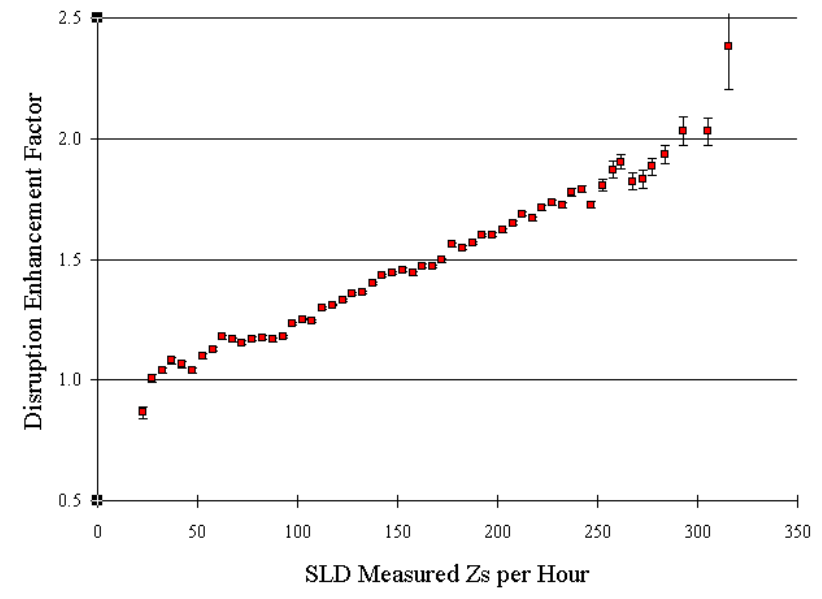

Figure 1: Measured disruption enhancement factor, $\mathrm{H}_{\mathrm{D}}$, plotted versus SLD measured Zs per hour.

\section{COMPARISON WITH THEORY}

The theoretical prediction for $\mathrm{H}_{\mathrm{D}}$ was calculated from the measured beam parameters using Chen's formalism for quasiflat beams as given in section 2 . The beam intensity was measured by toroids at the entrance to each final focus. The transverse beam size was taken from the most recent beam-beam deflection scans. The bunch length was measured infrequently and then continuously monitored for stability with microwave bunch length monitors in the linac and final focus [12]. The longitudinal distribution of events recorded by the SLD provided a cross check of the overlap of the two bunch lengths. The measured disruption enhancement agrees extremely well with the theoretical value as shown in Figure 2 by the close agreement between actual SLD event rates and those calculated including the theoretical estimate of disruption.

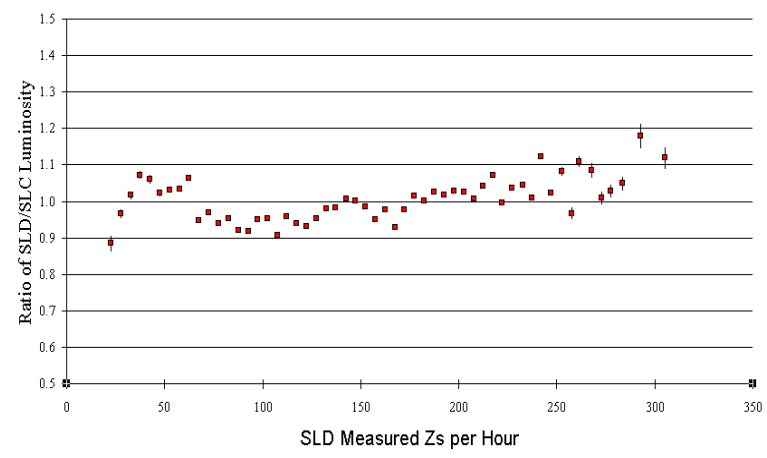

Figure 2: Ratio of measured SLD luminosity to calculated SLC luminosity including the theoretical estimate of disruption.

\section{CONCLUSIONS}

A significant luminosity enhancement from beam-beam disruption was observed for the first time at the SLC during the 1997-98 run, with peak values in excess of $100 \%$. The measured disruption was in agreement with theoretical predictions over a wide range of machine parameters. The improved SLC performance which led to this result was due to the hard work and dedication of many people, including the operations and maintenance staff and physicists from Accelerator Research and SLD. Their efforts are gratefully acknowledged by the authors.

\section{REFERENCES}

[1] R. Hollebeek, "Disruption Limits for Linear Colliders", Nucl. Instrum. Meth. 184, 333 (1981).

[2] P. Chen, "An Introduction to Beamstrahlung and Disruption", Frontiers. of Particle Beams 296, (1988).

[3] K. Yokoya, KEK Report 85-9 (1985).

[4] D. Schulte, "Beam-beam Simulations with GUINEA-PIG", CERN-PS-99-014.

[5] P. Chen and K. Yokoya, "Disruption Effects From The Interaction Of Round E+ E- Beams", Phys.Rev.D38, 987 (1988).

[6] P. Chen, "Disruption Effects from the Collision of Quasiflat Beams," PAC 93, Washington, DC, (1993).

[7] P. Emma, et al., "Limitations of Interaction Point Spot Size Tuning at the SLC," PAC 97, Vancouver, Canada, (1997).

[8] P. Raimondi, et al., "Disruption Effects on the Beam Size Measurement", PAC95, Dallas, Texas, (1995).

[9] P. Raimondi, et al., "Recent Luminosity Improvements at the SLC," EPAC 98, Stockholm, Sweden, (1998).

[10] L. Hendrickson, et al., "Luminosity Optimization Feedback in the SLC," ICALEPCS 97, Beijing, China, (1997).

[11] F.J. Decker, et al., "Longitudinal Phase Space Setup for the SLC Beams," PAC 97, Vancouver, Canada, (1997).

[12] F. Zimmermann, et al., "An RF Bunch Length Monitor for the SLC Final Focus," PAC 97, Vancouver, Canada, (1997). 